\title{
Correction to: Gray matter volume reduction with different disease duration in trigeminal neuralgia
}

\author{
Shanshan Shen ${ }^{1,2} \cdot$ Huifeng Zheng ${ }^{3}$. Jianwei Wang ${ }^{4}$. Wenchang Guo ${ }^{5}$ Xiaowan Guo ${ }^{1,2} \cdot$ Hong Ji $^{1,2}$. \\ Shuqian Zhang ${ }^{1,2} \cdot$ Yingmin Chen $^{1,2} \cdot$ Gaofeng Shi $^{1}$
}

Published online: 13 December 2021

(c) The Author(s), under exclusive licence to Springer-Verlag GmbH Germany, part of Springer Nature 2021

\section{Correction to: Neuroradiology (2021) \\ https://doi.org/10.1007/s00234-021-02783-y}

In the original publication "Gray matter volume reduction with different disease duration in trigeminal neuralgia", the annotation on Figs. 1, 2, and 3 were incorrect. The figures are now corrected with the correct notations.

The original article has been corrected.

Publisher's note Springer Nature remains neutral with regard to jurisdictional claims in published maps and institutional affiliations.

The original article can be found online at https://doi.org/10.1007/ s00234-021-02783-y.

Gaofeng Shi

447546952@qq.com

1 Department of Radiology, Fourth Affiliated Hospital of Hebei Medical University, Shijiazhuang, Hebei, China

2 Department of Radiology, Hebei General Hospital, Shijiazhuang, Hebei, China

3 Department of Radiology, Shexian Hospital, Handan, Hebei, China

4 Department of Radiology, Xianghe Hospital, Langfang, Hebei, China

5 Department of Neurosurgery, Hebei General Hospital, Shijiazhuang, Hebei, China 